\title{
Transforming Haptic Storyboards into Diagrammatic Models: The Scene2Model Tool
}

\author{
Elena-Teodora Miron \\ University of Vienna \\ elena@dke.univie.ac.at
}

\author{
Christian Muck \\ University of Vienna \\ christian.muck@univie.ac.at
}

\author{
Dimitris Karagiannis \\ University of Vienna \\ dimitris.karagiannis@univie.ac.at
}

\begin{abstract}
Haptic storyboarding tools supporting storytelling as a Design Thinking approach, enable early exploration and validation of design alternatives regarding services, new product (features), innovative processes and disruptive business models. They do however not communicate the exact meaning and the importance of each object nor do they show relationships between them. Yet when aiming to materialize an innovative idea these aspects need to be unambiguously described.

Diagrammatic models play an essential role here as they capture different aspects of the problem. When computed by means of software they also explicitly show details which in haptic storyboards users implicitly fill with their own world-understanding, thus fostering a clear and transparent representation of the problem space. In addition, diagrammatic models can be enriched by semantics and subsequently be machine-queried, -analysed and -processed.

The paper at hand shows the DIGITRANS project approach for an automated transformation of haptic storyboards into diagrammatic models and their provision in a computer-aided design environment.
\end{abstract}

\section{Introduction}

Digitization greatly challenges small and medium enterprises (SMEs) to be more innovative in their product/service offering as well as in their business models in order to remain competitive. In the exemplary case of a German SME, who was previously a producer of industrial ventilation systems, the price-driven competition from South-East Asia induced a steep business decline. Competition on product level was no longer feasible, as product features are quite standardized and easy to replicate. Thus the company transformed its business model by offering "air-as-aservice". This meant that industrial customers no longer paid for the product, i.e. ventilators, but for the service, i.e. the guarantee the firm provides, monitors and sustains an air quality corresponding to the respective standards in industrial settings.

To achieve this result the company had to a) tackle a problem space which had yet to manifest ("air-as-a-service"), b) solve a complex and diffuse new problem (the ecosystem for installing, operating and continuously monitoring as well as maintaining the ventilation system), c) integrate new actors (urban planners, construction companies, architects, software engineers) in the service design process and d) foster creativity and innovation in this new setting [1].

Requirements for the work on the innovative service and business model transformation within the company included a) collaborative design work of a multidisciplinary team, which was not usually collocated, b) the need for ongoing and asynchronous input into the project as not all ideas occur during a workshop, c) the need to share the project with distributed teams of different domain-experts, d) the need to access domain-specific views of the innovative service, beyond the big picture, in order to provide information on specific aspects of the solution and e) the need to integrate all stakeholder inputs in the evolution of the innovative solution and validate them against the original value proposition.

While Design Thinking concepts and tools like personas, strategy map, customer journey, empathy map etc. are helpful to foster ideation, teamwork and communication in early design phases and collocated environments, little research could be found on how to effectively support distributed multidisciplinary design teams during an innovation and transformation project. [2] propose a media model-based framework for team-based design thinking. While the approach incorporates diagrammatic models and Design Thinking, its aim is to explore how much Design Thinking is incorporated in software engineering tools and not on how tools can support the Design Thinking process. [1] propose an agile and participatory framework for social software engineering where they 
combine physical prototypes with digital artefacts to enable a better communication within the design team while at the same time maintaining the advantages from physical prototypes. However, the integration of distributed design teams is not considered. [3] create a computer-processable tool for design team interactions aiming to capture communication in real time and thus improve the overall communication process in complex and distributed project teams. Yet the focus is solely set on the communication aspect of the design process. [4] address distributed teams and the use of tangible means (e.g. sticky notes). Their proposed solution includes tele-boards, which digitize the complete Design Thinking process and tools, thus losing the advantages which tangible objects bring in the design process. [5] propose the media model approach to assess appropriate prototyping and modelling approaches in different stages of the Design Thinking process. Their research found that diagrammatic models, specifically business process management (BPM), are highly abstract, highly resolved and made available in media that restricts collaboration. To foster user involvement they argue for tangible artefacts, like prototypes of BPM classes. In line with [5] the paper at hand aims to explore:

How can tangible objects and abstract IT-artefacts be combined during the Design Thinking process to support the work of multidisciplinary, distributed design teams?

The research at hand was done in the context of DIGITRANS (cf. [6]), an EU-funded project, which delivers a Design Thinking-based method and a set of tools to support the digital transformation process of SMEs.

The remainder of this paper is structured as follows: Chapter 2 provides some background on storytelling and storyboarding in the context of Design Thinking and diagrammatic models as well as the research methodology applied. Chapter 3 presents the proposed modelling method and the Scene2Model tool. The tool and process evaluation results from a series of workshops are described in Chapter 4, while Chapter 5 concludes the work with an outlook on future activities.

\section{Background}

\subsection{Research methodology}

The work presented here follows the Action Design Research Method proposed by [7]. The initial problem formulation at the basis of our research was done through interviews with practitioners from the health care, creative industries and information and communication (ICT) domains in seven different European regions, where DIGITRANS project partners are located. The insights obtained were used to define the DIGITRANS method (cf. [8] as well as to research existing Design Thinking tools. The research question was formulated after exploring existing technologies and prior research.

Subsequently an initial design of the IT artefact proposed to solve the research question was conceptualized in the form of a modelling method and the Scene2Model prototype was implemented using the ADOxx [9] meta-modelling platform. A first evaluation was performed in small group workshops with selected organisations involved in problem finding as well as with other stakeholders. Evaluation was done using focus groups and questionnaires. The results of this initial evaluation are presented in Chapter 4 of this paper. Currently the input we received from organizational use is incorporated in a new design cycle. By October 2018 a larger number of organizations in different regions will use the second prototype in their context for further evaluation. This process is expected to yield several iteration cycles until May 2019. The evaluation questionnaires are currently being extended to better reflect the organizational context as well as the specific design team requirements. In parallel research work has been undergoing to better understand how technologies (like text recognition, ontologies etc.) can be integrated into the Design Thinking process to aid the innovation work of design teams. On a theory level research is ongoing on the use of diagrammatic models and haptic design artefacts in the media-models framework [5].

\subsection{Storyboards as a visualization tool for innovation and design}

One of the approaches DIGITRANS proposes for the innovation work of multidisciplinary design teams is storytelling. In an organizational setting storytelling can be used to observe and understand the explicit and implicit behaviour of (potential) customers [10], understand meaning-based needs as an innovation enabler [11], as well as a tool for strategic decision making [12]. Because problems faced in the digital transformation process tend to be complex and vaguely defined developing a story around them makes them easier to grasp.

Storyboards are a visualization tool which displays the key moments of a story, synthesizing them in a cohesive and illustrative flow [13]. The simplicity of storyboard sketches or objects and the light annotation is meant to encourage discussion, team collaboration 
[14] and enables designers to explain events, to identify logical errors in the story flow or present results [15]. Additionally, storyboards can be used to create prototypes for early solutions and test hypotheses [10]. However, research has shown that when directed at group work, like in innovation, storyboarding tools work best when all participants can contribute equally to the sketches and are not restricted by single-user interfaces, like a computer screen [15]. Additional research posits that using haptic storyboards where designers are able to touch and manipulate the objects provides an immersive experience and activates the brains most effective learning mechanism [16].

Although storyboards are easy to use and intuitively understandable, the only possibility to annotate them and add information is by attaching handwritten notes to the figures themselves. There is also, currently, no possibility to connect the different objects with each other. Thus the common understanding of the object positioning and meaning remains with the team who has developed them. This is a problem, because Design Thinking workshops are held in small groups, which means that only a few members of the overall project team can be involved, while others may need to be informed subsequently. If not accompanied by an explanatory written narrative, the context of the developed solution will be difficult to understand by non-team members. But long textual descriptions often hinder the usage of the generated information.

The results of haptic storyboards are also static. Their adaptation is often a non-trivial but necessary task, because sometimes specific aspects of a project are evolving during its implementation and it would be beneficial to capture these changes consistent to the initial documentation of the requirements.

Currently there is also no possibility to reference from the storyboard (panels) to a specific enterprise resource (e.g. existing capabilities, processes, etc.), which introduces a break in the process of transforming the innovative solution prototype into an operational product/service.

Transforming the haptic storyboard by means of an automated import into computer-aided diagrammatic models and facilitating semantic enrichment (e.g. by adding properties from domain ontologies) can help increase the information transparency, model consistency and clarify the meaning for model users. More formal abstractions (e.g. business process models, business capabilities, elements of enterprise architecture) help connecting the innovation to its enterprise context. The machine-readable semantics enable additionally automated mechanisms for design assessment and decision making.

\subsection{Storyboards in Design Thinking}

The Design Thinking process as described by [17] incorporates five distinct phases: empathizing, defining, ideation, prototyping and testing. Stories and storyboards can be used in each phase for different purposes.

The empathize mode is the work to understand people in the context of the design challenge [17] and aids designers in defining value propositions for the customer [11].

Sketching as an ideation technique can be done via storyboards, similarly to prototyping and testing. In an ideation context storyboards are useful to pre-structure story information, identify key events of the story, and visualise the logical flow of the information [18]. While working collaboratively the innovation team discusses the most important goals of the solution and they derive key value propositions, without describing the features and capabilities necessary to realize them [11].

During prototyping storyboards are used to generate a first solution design. Users and designers can engage in play, explore boundaries, test hypotheses and ultimately develop the best story for the new solution [10]. Similarly, storyboards are a quick and valuable means to test the convergence between user needs and the proposed solution.

\subsection{Diagrammatic models as a link between storyboards and enterprise models}

Diagrammatic models are, in our understanding, abstract representations aiming to reduce complexity by employing a specific type of representation, i.e. diagrams. They are used for a purpose, e.g. improving machine and human understanding, analysing or communicating properties of a system under study, and comply with a formal meta-model. Diagrammatic models are used for externalisation of knowledge and machine processing [19].

If deployed as a tool, diagrammatic models derived from a meta-model are Design Science artefacts comprising several building blocks [20]: a) a modelling language containing the notation, syntax (language grammar and constrains) and semantics (the domain) and b) mechanisms and algorithms to provide model-based functionality (like generation, simulation, model transformations, interactivity, automation, etc.) [19].

Diagrammatic models were used to overcome the shortcomings of the haptic storyboarding tools, as described previously, and enrich these with information and functionality aiming to facilitate 


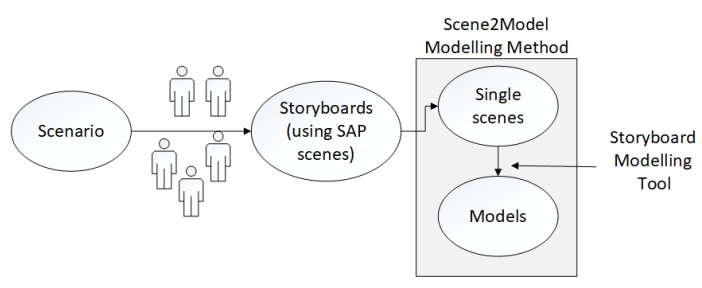

Figure 1. Visualization of the Scene2Model process

an easier integration into the context of a specific innovation and transformation project.

The conceptualization and implementation of the tool in the ADOxx platform [9], as well as the automatic transformation process between haptic objects and models are presented in the following chapter.

\section{The Scene2Model tool: Conceptualisation and proof-of-concept}

We propose a solution aiming to provide a computer-aided design environment which incorporates the benefits of haptic storyboards (e.g. physicality, extendability, intuitiveness, ease of use, orientation to co-creation and innovation) with diagrammatic models (e.g. abstraction, semantic enrichment, machine-processability), while at the same time automating as much of the transformation process between physical world and information system as possible to lower usage barriers.

The participants of the Design Thinking workshops use the storyboarding method. However, the created results can now be automatically transformed into a digital model, which then can be used and enriched in the modelling tool. In figure 11 the high-level process behind the Scene2Model approach is shown. A group of participants creates storyboards from their scenario, using SAP Scenes. Afterwards, each scene is imported into the Scene2Model modelling tool, where a diagrammatic model is created automatically.

An example on how such a transformation can be done is given in section 3.2 .

\subsection{Conceptualization of the Scene2Model method}

To achieve the desired integration a Design Thinking method, an enterprise modelling language and a domain ontology must be chosen (see Fig. 2) first. In our case we used storyboards for the former and business processes (BPMN) for the latter. The ontology created was general purpose.

The approach can be used for any Design Thinking

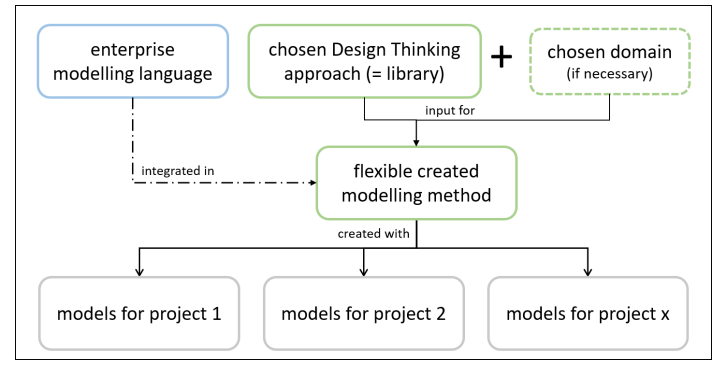

Figure 2. Conceptual framework of the Scene2Model method

method which complies with the minimal characteristics of a modelling method (e.g. Business Model Canvas, Customer Journey) and for most enterprise modelling languages and ontologies.

The objects of the selected Design Thinking method must be abstracted, so that the concepts used can be represented by the modelling method. For information on how modelling methods can be created and which aspects must be considered see for example [20, 21]. Depending on the chosen Design Thinking method, it is possible that a domain-specific context is necessary, which must be added if the proposed framework is used. One example for a domain sensitive approach would be the health care domain. The domain-specific vocabulary and concepts are important, as there must be a physical representation in form of haptic objects as well as usable concepts in the modelling tool for the innovation team to be able to use them in workshops.

To support the embedding of the created knowledge during a Design Thinking workshop and its created artefacts into the context of the enterprise, we suggest to integrate one or more enterprise modelling languages into the created modelling method and the computer-based modelling tool. This could be especially helpful, if the enterprise already uses diagrammatic modelling for other aspects of its business. Linking the Design Thinking models with enterprise models can be done during the workshop or afterwards. The modelling languages, which are used should increase the captured knowledge and in the ideal case should be integrated with an already existing pool of models, which is already available in the company.

For the models in the Scene2Model method a meta-model was created to define the different haptic objects. Using meta-modelling allowed us to group different modelling objects together and define properties for these groups or classes. Each class possesses multiple graphical presentations, which are defined by the Type of an object. Type is a property, which is available for every class, just like the properties Name and Description. The first one defines an identifier 


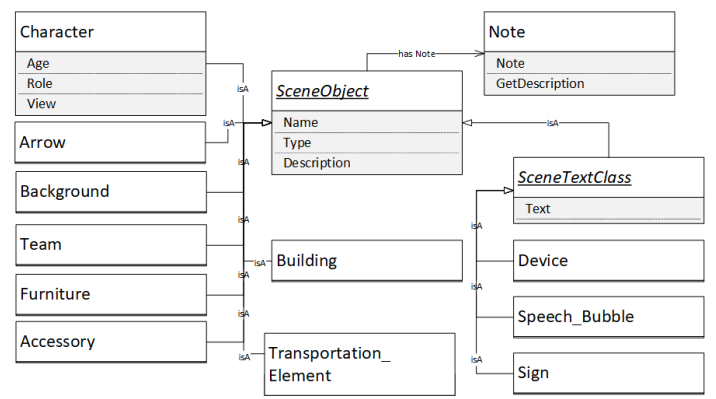

Figure 3. Visualization of the Scene2Model meta model

for a modelling object and the second one holds a natural language description. Classes with a Text property, show this text directly in the diagrammatic model. The class Character also implements the properties Role and Age, which can be used to specify humans in the modelled scenario. A graphical representation of the meta-model can be seen in figure 3 .

An ontology is used to map the IDs, from the tag recognition to modelling objects. Therefore, the information from the meta model is saved in the ontology and enhanced with a TagID property.

\subsection{Proof-of-concept: The Scene2Model tool}

The Scene2Model tool is a proof-of-concept prototype implementation of the proposed method. The goal of the implementation was to explore how an automatic transformation from the (often) physical Design Thinking methods to digital diagrammatic models can be done and to have a tool to collect first impressions on how such a process is received by potential users.

For implementing the prototype, we chose the SAP Scene (cf. [22]) storyboarding approach as the Design Thinking method. SAP Scene uses paper figures to visualize the key moments of the customer's interaction with the company's product or service. An example for such paper figures can be seen in figure 4. The figures are available in different downloadable domain-specific sets on SAP's homepage. Every key moment is represented by one scene, which can be then combined to storyboards. SAP Scenes was chosen because they offer a wide range of different and meaningful free figures.

The described meta model (cf. section 3.1) was implemented in the ADOxx meta-modelling platform, which supports the definition of classes and their properties, the implementation of mechanisms as well as the use of a built-in modelling toolkit for the created models.
One mechanism implementation is used to trigger the automatic transformation directly from the ADO $x x$ modelling tool. The data is gathered from an external application, which provides IDs of the paper figures and their position. Using these IDs additional information is read from an ontology and the diagrammatic model is created. A more detailed description of the Scene2Model implementation can be found later in this section.

The focus of the Scene2Model approach is on the automatic transformation of the tangible paper figures of SAP Scene into the digital model. Therefore, tags were attached to each paper figure. For an example see figure 4 on page 6 The advantage of those tags is that they can be recognised very efficiently by software. But not only the tag itself can be identified, the application is also able to determine the position in form of coordinates of a tag. Each tag represents one integer value, which then can be mapped to an object in the modelling tool. The integer value is an ID for the tag and each tag has a different and unique ID.

Therefore, from the tags alone an integer value and the position can be retrieved. The additional information needed for mapping the tag ID of the haptic figures to their corresponding modelling object is taken from an ontology. It contains information about what classes of objects are available for the modelling method and which properties can be assigned to the modelling objects. An ontology further allows to save semantic information, which can then be queried. The results can be automatically included in the modelling tool via services.

The implementation of the prototype itself consists of two applications. One is the modelling tool, which was implemented using the ADOxx meta-modelling platform. The other is the tag recognition software, which uses the ArUco library. The tag recognition software extracts the IDs, calculates the coordinates and sends the information to the modelling tool. This maps the IDs to the corresponding modelling objects and creates the diagrammatic models. The user is then able to change the properties of the objects, rearrange the model or add/delete modelling objects. The tag recognition software runs on a Linux-based operating system (Fedora for the prototype) and ADOxx is a Windows application.

Two settings were implemented for the Scene2Model prototype. One was designed for stationary and the other for a mobile usage. The stationary version uses a transparent table top and the tags on the paper figures faces downwards, in the direction of the table top. The camera for the recognition software is placed beneath the table and 
monitors the tags. In this scenario fixed markers are positioned on the table top (also facing downwards) and the position of the figures is calculated relatively to them. Using the fixed tags, the calculation of the tag coordinates is independent from the position of the camera. This allows the size of the table top to vary and is mostly dependent on the light conditions and the quality of the camera. The recognition software continuously sends the IDs and coordinates to the modelling tool, which can be fetched at any time by the user.

The mobile setting contains an A4-sized sheet, on which the figures are placed. In this scenario, the tags face upwards, so that the sheet can be put on any straight surface. Then a picture of the scene is taken, e.g. on a mobile phone, and the coordinates of the tags are calculated relatively to their position in the picture. In this setting the environment is easier to establish however the pictures, which are the basis for the automatic transformation, must be taken manually. Further, as the position is dependent on the size of the picture, it cannot be easily used with a bigger size than A4.

As last step, an enterprise modelling language was chosen, which should help to increase the captured knowledge of the used Design Thinking method. A single scene captures a static point in the user story and only through their combination can a dynamic behaviour be derived. Therefore, the BPMN business process modelling language was added to the Scene2Model method, to allow the capturing of dynamic aspects in a scene. Furthermore, BPMN is a well-known modelling language, which is already used in many companies. For the usage in the scene models, a process object was added, which visualizes one process. The object itself can be linked to a BPMN process model, which captures the dynamic aspects. Such a link is not mandatory. Initially the process object can be inserted only with a name and the model itself can be added later on. The processes can be connected to objects of a scene, to signal their togetherness.

A storyboard model and a process map can be automatically generated to allow a big picture overview for the design teams. The storyboard model links to the different scenes. The process map shows all process objects used in a single model.

The Scene2Model modelling tool and the tag recognition software can be downloaded from the OMiLAB project page for free. The project can be found under: http://austria.omilab.org/ psm/content/scene2model/info.

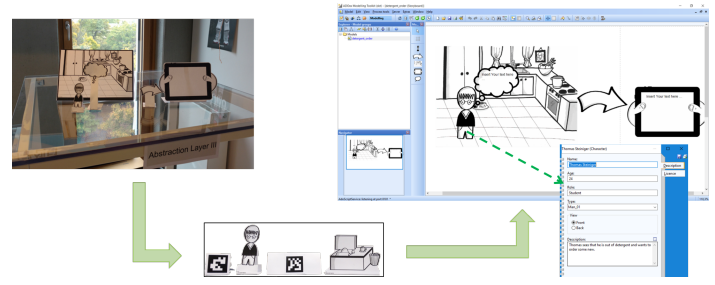

Figure 4. Visualization of the Scene2Model approach [23, p.4]

\section{Evaluation of the Scene2Model approach}

The Scene2Model approach is a proof-of-concept prototype implementation. To collect a first evaluation on how the approach and tool are accepted by potential users we conducted several small workshops with testing groups, where we gathered feedback using questionnaires and focus groups. This section will introduce the structure of the workshops, the questionnaire and the evaluation results.

\subsection{Case study design}

The group which tested the Scene2Model tool included several types of test persons. Half were professionals, either from industry or academia, and the other half were graduate students from Business Administration or Information Systems programs. In a series of eight workshops a total of 66 persons used the tool and gave their feedback via questionnaires. Out of the 66, 31 were students, 22 industry professionals and 8 academic staff. On five of the returned questionnaires the professional affiliation was missing.

Each workshop lasted for one and a half hours. Participants were given a short introduction to Design Thinking with focus on storytelling and storyboarding as well as on how to use the Scene2Model tool.

In a moderated session the teams co-created their scenarios using the haptic paper figures of SAP Scenes. Each finished scene was automatically imported into the modelling tool and after all scenes were completed, the whole group adapted and enhanced the digital models. A visualization of this process can be found in figure 4 To do so, the modelling tool was shown with a projector, one participant was assigned to operate the tool and all participants gave their input. The workshop moderator guided the discussion and provided technical support where necessary.

One important premise for the workshop was, that the participants had a scenario, which they could explore and discuss with the given haptic and computer-supported tools. Some of the students 
integrated their workshop in another course, so that they already started with a familiar scenario. For the other participants, a short scenario was prepared. Thereby it was important that the topic is well-known and broad enough, so that every workshop group can find a (sub-)scenario, on which they can focus and discuss the proposed solutions in detail.

The overall topic of the given scenario was smart mobility. The scenario describes the journey of a manager, who arrives at her home airport and must spontaneously join a meeting at her office. Even though it is her home town, she does not have in mind, which route is the fastest. There are also problems with the ticket machine, which costs her valuable time. After the meeting she has to perform follow-up work, even though she is getting hungry and does not know if she has something to eat at home. To summarize the trip, she encountered different problems, like not knowing the fastest route to the office building, no taxi was available and she does not know what she can cook at home as she does not remember what ingredients she has.

The description of the scenario itself was one A4-page long and the participants could choose on which (sub)-aspect of the problem they wanted to focus. The goal was to analyse the trips from the customer's perspective and find innovative solutions. How the customer interacts with the different solution ideas should be analysed using the haptic figures and the digital model.

\subsection{Acquisition of information}

The authors' goal during the workshops was to get feedback on the overall idea of the Scene2Model approach and on the prototypical implementation. The feedback should on one hand give an approximation about how useful the approach is deemed by the participants and on the other hand which improvements can be made. The acquisition of the information was based on three pillars. One was a questionnaire, which was given to each participant. The second one was a focus group at the end of each workshop. Finally, the participants were observed during the usage of the Scene2Model tool.

Because the major part of the Scene2Model approach is the computer-based tool support, it was decided to base the questionnaire on the System Usability Scale (SUS) (cf. [24, 25]). The usage and benefits of haptic figures was outside the scope of our evaluation. The SUS questionnaire is composed of ten questions and offers a short way to evaluate the usability of an information system. SUS was chosen because it is an established questionnaire, where evaluation guidelines and result interpretations are available. On the other hand, the Scene2Model approach is more than a computer-aided tool. It contains haptic figures, an automatic transformation and a creative and collaborative based approach. Therefore, we expanded the ten questions with three additional ones. In the end, our questionnaire consisted of the following thirteen questions or statements:

1. I think that I would like to use this system frequently.

2. I found the system unnecessarily complex.

3. I thought the system was easy to use.

4. I think that I would need the support of a technical expert to be able to use this system.

5. I found the various functions in this system were well integrated.

6. I thought there was too much inconsistency in this system.

7. I would imagine that most people would learn to use this system very quickly.

8. I found the system very cumbersome to use.

9. I felt very confident using the system.

10. I needed to learn a lot of things before I could get going with the system.

11. I think the haptic figures helped the exploring and discussing of the found scenarios.

12. I think the processable and enriched digital representation helps to use the results later in the implementation of found services/products.

13. A simple picture of the created scenes is more helpful than the digital model.

The first ten questions were taken from SUS (cf. [24], while the other three focused on the haptic figures and the estimation of the usefulness of the generated digital model. For all the thirteen questions, the user had to check one of five boxes, from strongly disagree to strongly agree. Therefore, every question could get between one (=strongly disagree) and five (=strongly agree) points.

Further, two open questions were embedded in the questionnaire to allow the participants to mention aspects which were not covered by the other thirteen questions. Therefore, the following two questions were added: 
- What do you think is the best aspect of this software, and why?

- What do you think needs most improvement, and why?

The focus group at the end of each workshop was used to get feedback on the structure of the workshop itself and a further evaluation on the Scene2Model approach. We wanted to know how participants liked the haptic paper figures and the automatic transformation. The questions and topics of the focus group were not as strict as in the questionnaire, but were based on authors' observations during the workshop and how discussion itself evolved.

\subsection{Results of the evaluation}

The first ten questions of the questionnaire were based on the computer-aided components of the Scene2Model approach and questions eleven to thirteen were mostly focused on the digital model and the haptic figures. The last three questions got above average rating. The twelfth question was about the enrichment of the digital models and if they could support a later implementation of the new-found service or product. This question got an average rating of approximately 4.17 points from maximum five. The eleventh question was focused on the usefulness of the paper figures and was rated with an average of approximately 4.03 points. The thirteenth question compared the usefulness of a digital model to taking a photo of the created haptic scene. This question got approximately an average of 3.23 points. These ratings indicate that the participants liked to discuss and explore their ideas with the means of haptic objects and found it beneficial to get an adaptable digital model for later usage. In the workshop they used the modelling tool to rearrange the modelling objects, add or delete some, define properties and add high-level processes to the created scenes. Regarding the preference of a digital model over a photograph the average participant was nearly indifferent between the two possibilities.

The first ten questions of the used questionnaire were taken from the well-known System Usability Scale approach and were also evaluated, using the approach from [25]. Using the proposed calculation we obtained a score of approximately 70 points. Using the classification from [26] our approach is set just beneath the threshold for the GOOD class. Therefore it is in the upper range of the $O K$ class. We concluded that the computer-aided part of the Scene2Model approach should be improved but is usable as it is.

The next part of the evaluation will be focusing on the qualitative questions from the questionnaire and the insights from the focus groups. Therefore, some of the individual expressions of the participants will be discussed. For this purpose we will consider the main questions, the authors wanted to answer with the evaluation.

4.3.1. How feasible is the Scene2Model approach for supporting creative Design Thinking processes? In the context of this question the focus was especially set on the following sub questions:

- Is the tool easy understandable for novices?

- How useful is the additional functionality, like the different export capabilities, importing of files and adaptation of the data?

- Can the tool support a connection between different domains (like e.g. Design Thinking with Business Process Management?

The questionnaire and the focus group discussion at the end of each workshop showed that most people were able to use the basic functionalities of the tool with only a short introduction. The transformation of haptic figures into a digital representation, adding/removing modelling objects, adapting the properties of the modelling objects and creating a process map or a storyboard are the functionalities which were introduced during the workshops. The possibility to share the models in an adaptable format were received positively by many participants and supports a collaboration between different stakeholders of common projects. A lot of information was generated during the discussion of the scenarios, using the haptic figures. It was deemed beneficial to add this information direct to the digital representation, through the adaptable properties of the modelling objects and textual notes. The average score of 70 points in the SUS questionnaire further underlines the usability of the tool. The straight-forwardness of the tool was one point, which was often highlighted in the first qualitative question. The feedback on the transformation functionality were mostly positive and many participants liked the way, how they could immediately start the adaptation of the digital model after the haptic scene was finished. In this context often an optimization of the transformation mechanism was suggested, like the positioning of the digital objects and the overall layout. For the digital representation itself, several participants wished for more drawing capabilities of the tool. Drawing in this context means, that they wanted basic geometrical forms (like circles, 
triangles, ...), the ability to change the colours of the object, rotate the objects and so on.

Regarding the combination of Design Thinking with Business Process Management, our observation and the feedback in the focus group showed that the combination of the created scenes with business process was a well-accepted feature. Especially the groups with their own scenario and therefore a more detailed idea of their use case and their goals, liked to add processes to their scenes. One suggestion for improvement in this context was the integration of already existing processes models, which could already exist in a company. Another idea was the extension to other (enterprise) modelling languages so that more different aspects of the companies can be added or integrated if they already exist. The approach of combining scenes with business process (or other enterprise modelling methods) seems promising. We learned that we needed to include question to this regard in further questionnaires and evaluations.

One negative aspect, which was often mentioned in the questionnaire and the focus group, was the limited set of figures for creating the tangible scenes. Many participants would have liked more figures to better express the scenario, as imagined by them. This was especially a problem by scenarios with a very narrow domain. From this feedback we learned that the figures should be tailored to the participants of a workshop and that it is important to have additional figures prepared, so that the participants are not limited in their creativity.

4.3.2. How useful is the combination of the digital and the haptic tools for the ideation stage? The results of the evaluation indicate that the haptic tools help to formulate and specify use cases. Participants, which already had an use case prepared, often found new aspects to it or changed smaller and bigger parts of it. In the qualitative questions and the focus group they stated that thinking through the use case step by step helped them to better understand the users perspective. These findings then led to changes in their primarily understanding of the scenario.

In the workshops the participants also adapted the automatic generated digital version together. This served as an evaluation of the defined haptic scenario and often lead to new ideas regarding the use case and the role of the customers in it. They used the modifying of the digital representation as an evaluation of the newly defined use case. In the feedback, the participants stated that the haptic tools supported their ideation and a mutual understanding of the scenario and the combination with the computer-based tool was also well-accepted by the participants. The goals and usage of the model differed from group to group. All groups used the digital model to add knowledge in form of text and properties of the modelling objects. Some groups additionally focused on the graphical representation and tried to make the digital pictures itself as expressive and appealing as possible. The focus of one other group during the workshop was mostly set on adding the needed business processes to the scene.

The acceptance of this approach could also be seen in questions eleven and twelve of the quantitative questions. Both questions got over four from five points. This supports the thesis, that a combination of digital and haptic tools can help in the ideation stage. The digital part often serves as a tool for rethinking the formed use case and supports a later usage of the generated knowledge.

One negative aspect that was mentioned by the participants was, that they had to install a program on their computer, if they wanted to use the models after the workshop. They had preferred an online tool, which can be used for interacting with the created models.

\section{Conclusion and future work}

In this paper an approach for an automatic transformation of the results from a haptic Design Thinking method into digital diagrammatic models was introduced. The approach was tested with a proof-of-concept prototype, which was evaluated with different testing groups. The idea of the automatic transformation and the prototype were well-received by the participants of the testing groups. Also, the usage of digital diagrammatic models was received as an improvement versus pictures or textual descriptions, which are often the results of Design Thinking methods.

As the conducted evaluation was meant to review the acceptance of the proposed approached and the first prototype, we are encouraged to further improve and enhance the approach. The request for online tool availability and the possibility to work remotely on the models indicated that teams saw value in having the models available at a later time and also being able to work independently on them.

Based on the feedback, we identified different aspects which demand further work. On the one hand the improvement of the functionalities of the Scene2Model prototype is important, so that the user satisfaction can be increased. On the other hand an analysis of other physical Design Thinking methods and how they can be automatically transformed into a digital model will be conducted. Further research is also necessary in how to ensure model consistency in 
distributed design teams as well as in how to integrate domain-specific views and enable checks for constraints and coherence in the overall scenario. Another interesting topic for the future is, which enterprise modelling language could be useful in the context of Design Thinking and how well they are accepted in the combination with Design Thinking.

This leads to the need to further improve the existing and the implementation of new prototypes and their evaluation in organizational contexts to contribute to the practical applicability of the Scene2Model approach in industry design environments.

Acknowledgement: The work presented here was funded by the Danube Transnational Program through the DIGITRANS (DTP1-193-1.2) project. This paper uses Scenes ${ }^{T M}$ by SAP AppHouse (https://experience.sap. com/designservices/approach/scenes),

licensed under CC-BY-NC-SA 4.0 International License (https://creativecommons.org/ licenses/by-nc-sa/4.0/).

\section{References}

[1] P. Newman, M. Ferrario, W. Simm, S. Forshaw, A. Friday, and J. Whittle, "The role of design thinking and physical prototyping in social software engineering," in Proceedings of the 37th IEEE International Conference on Software Engineering, pp. 487-496, Florence, Italy, 2015.

[2] A. Grosskopf, J. Edelman, M. Steinert, and L. Leifer, "Design thinking implemented in software engineering tools proposing and applying the design thinking transformation framework," 2010.

[3] M. Uflacker, T. Kowark, and A. Zeier, "An instrument for real-time design interation capture and analysis," in Design Thinking: Understand - Improve - Apply, pp. 131-145, Springer-Verlag Berlin Heidelberg, 2011.

[4] R. Gumienny, C. Meinel, L. Gericke, M. Quasthoff, P. LoBue, and C. Willems, "Tele-board: Enabling efficient collaboration in digital design spaces across time and distance," in Design Thinking: Understand Improve - Apply, pp. 147-164, Springer-Verlag Berlin Heidelberg, 2011.

[5] J. Edelman and R. Currano, "Re-representation: Affordances of shared models in team based design," in Design Thinking: Understand - Improve - Apply, pp. 61-79, Springer-Verlag Berlin Heidelberg, 2011.

[6] "Digitrans - digital transformation in the danube region." http://www.interreg-danube.eu/ approved-projects/digitrans. Accessed: 7-6-2018.

[7] K. Maung, O. Henfridsson, S. Purao, M. Rossi, and R. Lindgren, "Action design research," MIS Quarterly, vol. 35, no. 1, pp. 37-56, 2011.

[8] DIGITRANS CONSORTIUM, "Digitrans method for the digital transformation of smes." http:www. digitrans.me

[9] BOC Group, "Adoxx.org - a meta-modelling platform." http:wWw.adoxx.org
[10] M. Beckman, Sarah L.and Barry, "Design and innovation through storytelling," International Journal of Innovation Science, vol. 1, no. 4, pp. 151-160, 2009.

[11] M. Beckman, Sarah L.and Barry, "Innovation as a learning process: Embedding design thinking," California Management Review, vol. 50, no. 1, pp. 25-56, October 1st, 2007.

[12] S. Denning, The leader's guide to storytelling: mastering the art and discipline of business narrative. John Wiley and Sons, Inc., 2005.

[13] N. M. Yusoff and S. S. Salim, "A review of storyboard tools, concepts and frameworks," in Int. Proceedings of the LCT Conference, Part 1, LNCS8523, pp. 73-82, Springer, 2014.

[14] A. Malizia, A. Bellucci, P. Diaz, I. Aedo, and S. Levialdi, "estory: A visual storyboard system supporting back-channel communication for emergencies," Journal of Visual Languages and Computing, vol. 22, no. 2, pp. 150-169, April 2011.

[15] R. Walker, L. a. Cenydd, S. Pop, and H. C. Miles, "Storyboarding for visual analytics," Information Visualization Journal, vol. 14, no. 1, pp. 27-50, 2015.

[16] M. R. Bhalla, B. H. Vardhan, and A. V. Bhalla, "Haptic technology: An evolution towards naturality in communication," International Journal of Advanced Research in Computer Science, vol. 1, no. 4, pp. 97-103, 2010.

[17] Institute of Design Standford, "An introduction to desing thinking: Process guide." https: //dschool-old.stanford.edu/ sandbox/groups/designresources/ wiki/36873/attachments/74b3d/ ModeGuideBOOTCAMP 2010L.pdf

[18] L. Jeanne, "Innovative ways companies are using design thinking," Strategy and Leadership Journal, vol. 42, no. 2, pp. 40-45, 2014.

[19] D. Karagiannis, R. A. Buchmann, and M. Walch, "How can diagrammatic conceptual modelling support knowledge management?," in Proceedings of the 25th European Conference on Information Systems, pp. 1568-1583, AIS Electronic Library, 2017.

[20] D. Karagiannis and H. Kühn, "Metamodelling platforms," in $E C$-Web, vol. 2455, p. 182, 2002.

[21] D. Karagiannis, "Agile modeling method engineering," in Proceedings of the 19th Panhellenic Conference on Informatics, pp. 5-10, ACM, 2015.

[22] SAP SE, "Scenes - every great experience starts with a great story." https://experience.sap.com/ designservices/approach/scenes

[23] E.-T. Miron, C. Muck, D. Karagiannis, and D. Götzinger, "Transforming storyboards into diagrammatic models," in International Conference on Theory and Application of Diagrams, pp. 770-773, Springer, 2018.

[24] J. Brooke et al., "Sus-a quick and dirty usability scale," Usability evaluation in industry, vol. 189, no. 194, pp. 4-7, 1996.

[25] J. Brooke, "Sus: A retrospective," J. Usability Studies, vol. 8, pp. 29-40, Feb. 2013.

[26] A. Bangor, P. Kortum, and J. Miller, "Determining what individual sus scores mean: Adding an adjective rating scale," J. Usability Studies, vol. 4, pp. 114-123, May 2009. 